\title{
A set of 150 pictures with morphologically complex English compound names: Norms for name agreement, familiarity, image agreement, and visual complexity
}

\author{
Niels Janssen • Petra E. Pajtas • Alfonso Caramazza
}

Published online: 18 March 2011

(C) Psychonomic Society, Inc. 2011

\begin{abstract}
We present a set of 150 pictures with morphologically complex English compound names. The pictures were collected from various sources and were standardized to appear as grayscale line drawings of a fixed size. All the compounds had two constituents and were primarily of the noun-noun type. Following previous studies, we collected name agreement (percentage and $H$ ), familiarity, image agreement, and visual complexity norms, as well as frequency estimates for the whole compound word and its first and second constituents. These pictures and their corresponding norms (available from the Psychonomic Society's supplemental archive) are a valuable tool in the study of the morphological representation of complex words in language processing.
\end{abstract}

Electronic supplementary material The online version of this article (doi:10.3758/s13428-011-0065-0) contains supplementary material, which is available to authorized users.

N. Janssen

University of La Laguna,

Tenerife, Spain

P. E. Pajtas $\cdot$ A. Caramazza

Harvard University,

Cambridge, MA, USA

P. E. Pajtas $\cdot$ A. Caramazza

Center for Mind/Brain Sciences, University of Trento,

Trento, Italy

N. Janssen $(\bowtie)$

Grupo Neurociencia Cognitiva \& Psicolingüística, Dpto. de Psicología Cognitiva, Social y Organizacional, Facultad de Psicología, Universidad de La Laguna,

Campus de Guajara s/n - La Laguna,

38205 Tenerife, España

e-mail: njanssen@ull.es
Keywords Picture naming norms $\cdot$ Language Compounds $\cdot$ Morphology

\section{Introduction}

Much of the scientific progress in the field of cognitive psychology is due to the availability of standardized materials that allow for comparisons across studies and research groups. The set of standardized pictures collected by Snodgrass and Vanderwart (1980) has been particularly influential for researchers interested in the (neuro-) cognitive processes underlying word production (see, e.g., Goldrick, Folk, \& Rapp, 2010; Hocking, McMahon, \& Zubicaray, 2010; Strijkers, Costa, \& Thierry, 2010). One shortcoming of these norms is that the pictures generally elicit monomorphemic names and are, therefore, unsuited for researchers interested in the question of how morphologically complex words are produced. To our knowledge, there are no standardized picture sets that elicit morphologically complex names. This situation clearly limits progress in the study of the role of morphology in language production, as evidenced by the relative paucity of studies on this topic (exceptions are Badecker, 2001; Bien, Levelt, \& Baayen, 2005; Dohmes, Bölte, \& Zwitserlood, 2004; Janssen, Bi, \& Caramazza, 2008; Roelofs \& Baayen, 2002).

In the present study, we sought to standardize and collect norms for a set of 150 pictures with morphologically complex names. Specifically, all pictures had English compound names (e.g., windmill). Each compound had two major constituents, and for each compound, the second constituent was a noun. While the majority of the 150 compounds were of the nounnoun type $(n=128)$, there were also adjective-noun $(n=10)$ and noun-verb $(n=12)$ types. As in previous studies, for each picture, name agreement, familiarity, image agreement, 
and visual complexity norms were collected (e.g., Alario \& Ferrand, 1999; Cycowicz, Friedman, Rothstein, \& Snodgrass, 1997; Dimitropoulou, Duñabeitia, Blitsas, \& Carreiras, 2009; Sanfeliu \& Fernández, 1996; Snodgrass \& Vanderwart, 1980).

These norms have been shown to impact performance in a variety of tasks (see Alario et al., 2004, for an overview). Name agreement, which refers to the degree to which participants agree upon a name for a given picture, correlates negatively with picture-naming latencies (e.g., Lachman, Shaffer, \& Hennrikus, 1974; Vitkovitch \& Tyrell, 1995) and is unaffected by the frequency of the picture name (Lachman et al., 1974). Familiarity, which refers to the subjective familiarity with the concept that is depicted, has been shown to correlate negatively with lexical decision latencies (e.g., Gernsbacher 1984; Gordon, 1985). There is a discussion as to whether familiarity affects semantic or lexical processing (e.g., Balota, Pilotti, \& Cortese, 2001). Image agreement refers to the degree to which participants judge their mental image of an object to be similar to an actually presented object. Previous studies have shown that this variable is negatively correlated with picture-naming latencies (Barry, Morisson, \& Ellis, 1997). Finally, visual complexity refers to the complexity of the lining of an image and is thought to measure processes at early stages of picture processing. Previous research has either reported a negative correlation between visual complexity and picture identification (e.g., Ellis \& Morisson, 1998) or has not found this variable to be influential (e.g., Snodgrass \& Yuditsky, 1996).

The procedure of collecting the picture norms described below differed in an important way from the procedure used in previous studies (e.g., Alario \& Ferrand, 1999; Snodgrass \& Vanderwart, 1980). In previous studies, norms were typically collected in an untimed fashion; pictures were presented in a classroom setting, using a slide projector, to many participants simultaneously. Participants were then asked to write down their answers on a sheet of paper and were given ample time to prepare their responses (typically around $5 \mathrm{~s}$ ). A general problem with this approach is that the setting in which the norms are collected differs from the setting in which the pictures and their norms are typically used (i.e., timed, computerized experiments). Given that there is ample evidence for the influence of situational context on various cognitive processes (e.g., Godden \& Baddeley, 1975), we decided to elicit picture norms under timed circumstances that more accurately reflect a typical experimental setting. Specifically, participants were shown pictures on the computer screen and had to produce a response to the picture in less than $2 \mathrm{~s}$. This timed procedure has been used to collect name agreement norms (e.g., Snodgrass \& Yuditsky, 1996; Székely et al., 2003), but not for variables that consist of ratings such as familiarity, image agreement, or visual complexity. We directly addressed the degree to which timed norms are better predictors of timed picture-naming latencies than are untimed norms by examining a subset of the pictures for which both timed and untimed norms were available.

\section{Method}

Participants Ninety Harvard University students participated in the study. Thirty participants took part in the name agreement study, 29 took part in the familiarity study, and 31 took part in a joint image agreement and visual complexity study. All participants were native speakers of English. They received either $\$ 10$ per hour or course credit as compensation for their participation. Participants were all tested individually, not in a group setting.

Materials One hundred fifty pictures were selected from several sources (e.g., Google image search; ArtExplosion, 1998; Cycowicz et al., 1997). Selected images had the following properties: (1) All the images depicted objects (not actions); (2) all the images were simple line drawings (not actual photographs); and (3) in all the images, the object appeared by itself, without a background scene. In addition, object names had the following properties: (1) Object names were listed as compounds in the CELEX lexical database (Baayen, Piepenbrock, \& van Rijn, 1993; 12 compounds were not listed; see Appendix 1); (2) they had a maximum of two constituents, where the second constituent was always a noun; and (3) all compounds were of the noun-noun $(n=128)$, adjective-noun $(n=10)$, or verb-noun $(n=12)$ type. The objects were centered as 8-bit grayscale line drawings on a white background. The size of each image was $260 \times 260$ pixels.

Procedure The three studies used a timed and computerized version of the procedure outlined by Snodgrass and Vanderwart (1980). Norms were collected for name agreement, familiarity, image agreement, and visual complexity. However, instead of asking participants to write down their answers in response to pictures presented with a slide projector (e.g., Alario \& Ferrand, 1999; Snodgrass \& Vanderwart, 1980), we presented the pictures on the computer screen using DMDX experiment software (Forster \& Forster, 2003), and we collected the participants' keypresses or vocal responses (using a microphone).

To collect the name agreement norms, participants named the set of 150 pictures in a random order. Prior to the experiment, participants were told that they would see an object on the computer screen and that they should say aloud the first thing that provided an accurate description of 
the object. They were instructed to do this as quickly as possible. When they did not recognize the object or when they could not recall the object's name, they were told not to respond. They were also told that after a while, the object would disappear, and the object's name would appear on the screen. On each trial, the participants saw a fixation cross (700 ms), a blank screen $(200 \mathrm{~ms})$, the picture $(2,000 \mathrm{~ms})$, and the name the experimenter had intended for the picture $(1,000 \mathrm{~ms})$, followed finally by a blank screen $(2,000 \mathrm{~ms})$. Naming the 150 pictures took approximately $20 \mathrm{~min}$.

Familiarity, image agreement, and visual complexity norms were also collected using a computer. Participants in the familiarity task were told that they would see a picture presented on the computer screen and that they would have to rate "how usual or unusual the object is in your realm of experience." Familiarity was defined as "the degree to which you come in contact with or think about the concept" (Snodgrass \& Vanderwart, 1980). Participants were told to rate the concept itself, rather than the way it was drawn. The ratings used a 7-point scale, where 1 indicated a very unfamiliar object and 7 indicated a very familiar object. Participants were encouraged to use all seven ratings. Participants used the keyboard to press the number key that corresponded to their rating. On each trial, the picture remained on the screen for $2,000 \mathrm{~ms}$, after which the next picture was presented.

Norms for image agreement and visual complexity were obtained from one group of participants. These two norms were collected in a blocked fashion, where participants first rated all the pictures on image agreement and then on visual complexity. In the image agreement block, participants were told that they would have to judge how closely a given picture resembled their mental image of the object. They were told that they would see the name of an object on the computer screen $(3,000 \mathrm{~ms})$ and that they had to form a mental image of the object. After 3,000 ms, a picture of the object followed, and they were required to rate the degree of agreement between their mental image and the picture, using a 7-point scale (where 1 indicated low agreement between their image and the picture and 7 indicated high agreement). In the subsequent visual complexity block, participants were told that they would see the pictures again and that, this time, they should rate the complexity of each picture, using a 7point scale (where $1=$ very simple and $7=$ very complex). They were instructed to rate the complexity of the drawing ("the amount of detail or intricacy of lines in the picture," per Snodgrass \& Vanderwart 1980), rather than the complexity of the real-life object it represented. The trial structure in these two tasks was identical to the one used in the familiarity task.

In addition to the norms described above, lexical frequency estimates were collected for the names of the objects. For each object name, the surface and the first- and second-constituent lemma frequencies were computed on the basis of the frequency estimates listed in the CELEX lexical database (Baayen et al., 1993). Lemma frequency corresponds to the frequency of a word as it appears in all its inflectional variants (e.g., the lemma frequency of windmill would be the frequency of the word windmill plus the frequency of windmills). For the 12 compounds in the set that were not listed in CELEX, we assumed that the frequency was zero. Since these frequency estimates are typically used in studies investigating the morphological representation of words, it will be informative to examine the relationship between these estimates and the picture's norms.

\section{Results and discussion}

Picture names and norms are presented in Appendix 1 in alphabetic order. In addition, the pictures themselves are available as a compressed archive upon request from the first author or directly from the Psychonomic Society's supplemental archive. For each picture, the following information is presented: (1) an item number that is consistent across Appendices 1 and 2, (2) the most commonly used name, (3) the name as it is listed in CELEX, (4) the type of compound (e.g., noun-noun), (5) the two measures of name agreement (the statistic $H$ and the percentage), (6) the means and standard deviations for the familiarity, image agreement, and visual complexity norms, (7) the surface and first- and second-constituent frequencies, and (8) the average picture-naming latency obtained from the name agreement study. Appendix 2 provides a list of the alternative names given to each picture. Failures in the naming task are listed as NR (no response).

Following Snodgrass and Vanderwart (1980), name agreement was expressed in terms of the proportion of participants who produced the most common name for the picture and in terms of the metric $H . H$ represents a point-estimator for the distribution of the proportion of different responses given to a particular picture (i.e., its entropy; Shannon, 1948). $H$ therefore provides a useful measure that captures the degree to which participants were consistent in producing a name for a specific picture. $H$ can range from 0 to infinity, where values around 0 indicate perfect name agreement (all participants provided the same name) and larger values indicate more variation in the names given for a particular picture. It is important to note that decreasing values of $H$ indicate an increase in name agreement. The following equation was used to compute $H$ :

$H=\sum_{i=1}^{k} p_{i} \log _{2}\left(1 / p_{i}\right)$ 
where $k$ represents the number of different names given for a particular picture and $p_{i}$ denotes the proportion of participants who produced a particular name. In line with previous studies (e.g., Snodgrass \& Vanderwart, 1980), we eliminated no responses (NR) from the data set prior to the calculation of $H$, but not for the calculation of percentage of name agreement.

Table 1 lists the means and standard deviations, as well as Tukey's five-point summary (Tukey, 1977) for each of the following variables in our set: name agreement ( $H$ and percentage), familiarity, image agreement, and visual complexity. Also listed are the (log-transformed) surface frequency and first- and second-constituent frequency estimates from CELEX. Following Snodgrass and Vanderwart (1980), we use $H$ as the index of name agreement. Figure 1 represents the distribution of these variables, using histograms and density functions.

The measured name agreement has a low mean (0.95), and its distribution is skewed leftward, indicating that there are many pictures in the set with high name agreement (Fig. 1a, b). The bimodal distribution of the surface frequency variable (Fig. 1f) reflects the fact that in the CELEX database, frequency estimates for many compounds in our set are zero or close to zero. Since compounds are generally long words, it is expected that, on average, their frequencies are low; this pattern is expected on the basis of Zipf's law, which states that the frequency of occurrence of a word is inversely proportional to the length of the word (Zipf, 1932). However, it should be noted that not all compounds in our set have zero or close-to-zero frequency; the histogram in Fig. 1f shows that there is a reasonable amount of spread in surface frequency. In addition, significant surface frequency effects on picturenaming latencies have been found with pictures chosen from this set (Janssen et al., 2008).
As was previously stated, our procedure for collecting picture norms differed from the procedure used in previous studies: we collected norms under timed conditions, whereas in previous studies, participants' written and vocal responses to a picture were collected without time constraints (Alario \& Ferrand, 1999; Sanfuliu \& Fernández, 1996; Snodgrass \& Vanderwart, 1980). Following the argument in the introduction, one might expect the norms collected under timed conditions to be better predictors of timed picture-naming latencies than are norms collected under untimed conditions. Untimed norms were available for a subset of 28 pictures from the original Snodgrass and Vanderwart study. In order to assess the degree to which timed norms are better predictors of timed naming latencies, three different analyses were performed on the timed and untimed norms available for the 28 pictures.

First, Pearson correlations were computed between the timed and untimed norms to verify that both norming procedures captured similar underlying constructs. Correlations between timed and untimed norms were high (see Table 2), suggesting that our norming procedure captures similar underlying constructs as the untimed procedure. Second, linear models that contained only a timed or a corresponding untimed norm were fitted to the naming latencies from the name agreement experiment, using a standard item-based regression analysis (i.e., ordinary least squares). Except for visual complexity, both timed and untimed norms were significant predictors of naming latency (see Table 3). Importantly, however, the timed predictors were more accurate, because they accounted for a larger amount of variance (i.e., a larger adjusted $R^{2}$ ). Finally, item-based regression analyses examined whether linear models that contained a predictor corresponding to a timed norm plus a corresponding

Table 1 Summary statistics for all variables

\begin{tabular}{|c|c|c|c|c|c|c|c|c|}
\hline \multirow[b]{2}{*}{ Statistic } & \multicolumn{2}{|c|}{ Name Agreement } & \multirow[t]{2}{*}{ Familiarity } & \multirow{2}{*}{$\begin{array}{l}\text { Image } \\
\text { Agreement }\end{array}$} & \multirow{2}{*}{$\begin{array}{l}\text { Visual } \\
\text { Complexity }\end{array}$} & \multirow{2}{*}{$\begin{array}{l}\text { Surface } \\
\text { Frequency* }\end{array}$} & \multirow{2}{*}{$\begin{array}{l}\text { 1st Constituent } \\
\text { Frequency* }\end{array}$} & \multirow{2}{*}{$\begin{array}{l}\text { 2nd Constituent } \\
\text { Frequency* }\end{array}$} \\
\hline & $H$ & $\%$ & & & & & & \\
\hline$M$ & 0.95 & 67.50 & 3.69 & 5.1 & 3.8 & 2 & 6.52 & 6.41 \\
\hline$S D$ & 0.80 & 29.14 & 1.35 & 0.83 & 1.31 & 1.7 & 1.66 & 1.61 \\
\hline Median & 0.86 & 75.00 & 3.70 & 5.17 & 3.69 & 1.79 & 6.80 & 6.43 \\
\hline Min & 0 & 0 & 1.32 & 2.39 & 1.19 & 0 & 0 & 1.61 \\
\hline Max & 3.23 & 100.00 & 6.87 & 6.78 & 6.91 & 7.02 & 9.61 & 10.28 \\
\hline Q1 & 0.26 & 46.67 & 2.48 & 4.59 & 2.74 & 0 & 5.58 & 5.35 \\
\hline Q3 & 1.57 & 93.33 & 4.59 & 5.72 & 4.63 & 3.30 & 7.64 & 7.52 \\
\hline Skew & 0.56 & -0.70 & 0.23 & -0.58 & 0.27 & 0.45 & -0.80 & -0.17 \\
\hline
\end{tabular}

Note. Frequency taken from CELEX (Baayen, Piepenbrock, \& van Rijn, 1993). M, mean; SD, standard deviation; Q1, 25th percentile; Q3, 75th percentile; Skew, skewness (Joanes \& Gill, 1998)

*Log transformed values 
Fig. 1 Histogram and density functions representing the distribution of the variables name agreement $(\%$, panel $\mathrm{A} ; H$, panel B), familiarity (panel C), image agreement (panel D), visual complexity (panel E), log surface frequency (panel F), $\log$ first constituent frequency (panel G), and log second constituent frequency (panel $\mathrm{H}$ ). Rel. Frequency. relative frequency of occurrence
A

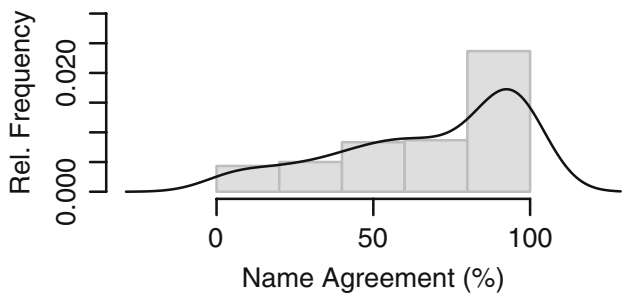

C

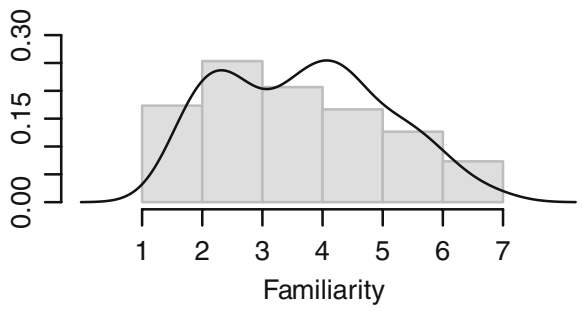

E

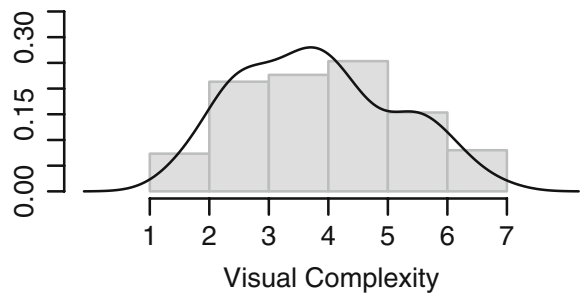

G

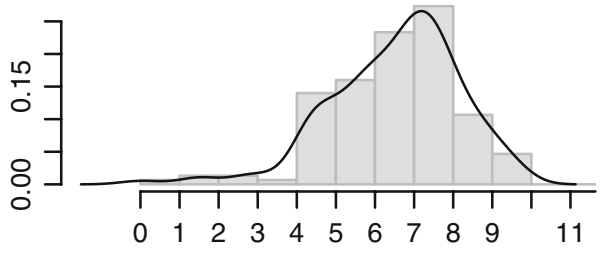

(log) 1st Constituent Frequency
B

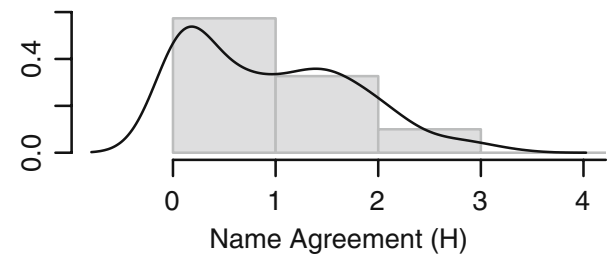

D

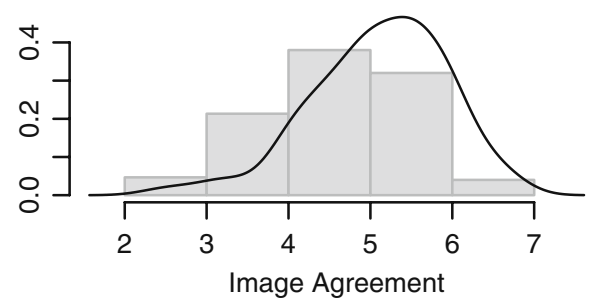

$\mathbf{F}$

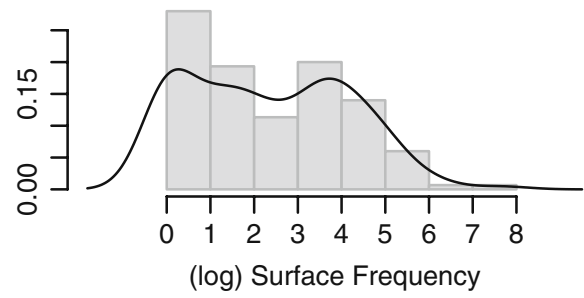

H

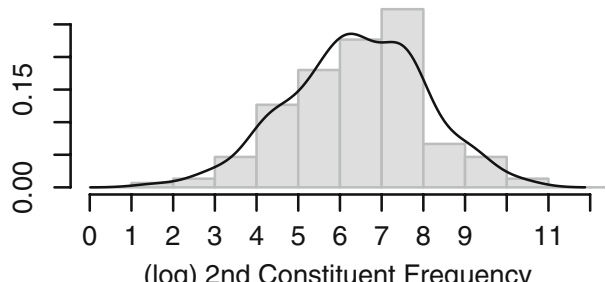

(log) 2nd Constituent Frequency
Table 2 Correlations for a subset of 28 pictures between timed norms collected here and untimed norms extracted from Snodgrass and Vanderwart (1980)

\begin{tabular}{ll}
\hline Norm & $\begin{array}{l}\text { Correlation Between Timed } \\
\text { and Untimed Norm }\end{array}$ \\
\hline Name agreement $(\mathrm{H})$ & $.58^{*}$ \\
Name agreement $(\%)$ & $.67^{*}$ \\
Familiarity & $.87^{*}$ \\
Image agreement & $.64^{*}$ \\
Visual complexity & $.69^{*}$ \\
\hline
\end{tabular}

*Correlation coefficients significant at $p<.01$ decorrelated untimed predictor were justified over models that contained only the predictor corresponding to the timed norm. Model comparisons using $F$ tests (e.g., Baayen, 2008) revealed no differences between these two models for each norm, suggesting that the presence of the decorrelated untimed predictor was unjustified in the model (all $F_{\mathrm{S}}<1$ ). Overall, these analyses show that, for the subset of pictures used here, the timed norms are more accurate predictors of naming latency than are the untimed norms. This makes it recommendable that future studies rely on a timed procedure for collecting picture norms.

Table 4 lists the intercorrelations of the norms collected for the 150 pictures in the present study. In addition, the 
Table 3 Overview of the $t, p$, and adjusted $R^{2}$ statistics obtained after fitting the name agreement ( $H$ and percentage), familiarity (FAM), image agreement (IA), and visual complexity (COMP) norms obtained using a timed procedure (present study) or using an untimed procedure (Snodgrass \& Vanderwart, 1980) to the average picture-naming latencies from the name agreement study. Each variable was fitted in a separate model

\begin{tabular}{|c|c|c|c|c|c|c|}
\hline & \multicolumn{3}{|l|}{ Timed } & \multicolumn{3}{|c|}{ Untimed } \\
\hline & $t(26)$ & $p$ & Adj. $R^{2}$ & $t(26)$ & $p$ & Adj. $R^{2}$ \\
\hline$H$ & 6.26 & $<.001$ & .59 & 2.51 & $<.02$ & .16 \\
\hline$\%$ & -7.21 & $<.001$ & .65 & -2.96 & $<.007$ & .22 \\
\hline FAM & -2.08 & $<.05$ & .11 & -1.00 & .24 & 0 \\
\hline IA & -3.79 & $<.001$ & .33 & -2.59 & $<.02$ & .17 \\
\hline COMP & -0.10 & .92 & 0 & -0.14 & .89 & 0 \\
\hline
\end{tabular}

correlations of these norms with the frequency estimates that are typically used in language-processing studies are computed. For completeness sake, the intercorrelations of these variables were also computed. Significant correlations are marked with an asterisk. Overall, the intercorrelations found between the norms obtained in the present study are similar to those from previous studies (Alario \& Ferrand, 1999; Sanfeliu \& Fernández, 1996; Snodgrass \& Vanderwart, 1980).

A strong negative correlation (-.88) was expected between the variables $H$ and percentage of name agreement (Table 4), since both variables measure a picture's name agreement. In addition, name agreement $(H)$ correlated somewhat negatively with familiarity $(-.26)$, indicating that pictures with a higher name agreement were also more familiar to our participants. There was also a negative correlation between name agreement and image agreement (-.49). This suggests that objects with a high name agreement were also judged to better match the mental images that participants had of these objects. In addition, there was a positive correlation between familiarity and image agreement (.29), suggesting that with increasing familiarity, there was a better match between a participant's mental image and the actual image; there was also a correlation between familiarity and complexity (-.20), indicating that pictures were judged to be more familiar when their visual complexity was lower. Finally, visual complexity ratings were correlated with the frequency of the second constituent (-.22), suggesting a relationship between the complexity ratings and the frequency of the compound's second constituent.

\section{Conclusion}

Our main motivation for the present study was to present a standardized set of pictures that elicit morphologically complex names. We collected name agreement, familiarity, image agreement, and visual complexity norms for 150 pictures with English compound names. It is our hope that the availability of these pictures and their corresponding norms will stimulate research into the role of morphology in language processing and that comparable norms will eventually become available in languages other than English.

Acknowledgements This research was sponsored by a Juan de la Cierva postdoctoral fellowship from the Spanish Government to N.J., and by Grant R01DC006842 from the National Institute on Deafness and Other Communication Disorders to A.C.

Table 4 Correlations among the variables collected for the 150 pictures in the present study

\begin{tabular}{|c|c|c|c|c|c|c|c|c|}
\hline \multirow[b]{2}{*}{ Statistic } & \multicolumn{2}{|c|}{ Name Agreement } & \multirow[t]{2}{*}{ Familiarity } & \multirow{2}{*}{$\begin{array}{l}\text { Image } \\
\text { Agreement }\end{array}$} & \multirow{2}{*}{$\begin{array}{l}\text { Visual } \\
\text { Complexity }\end{array}$} & \multirow{2}{*}{$\begin{array}{l}\text { Surface } \\
\text { Frequency* }\end{array}$} & \multirow{2}{*}{$\begin{array}{l}\text { 1st Constituent } \\
\text { Frequency* }\end{array}$} & \multirow{2}{*}{$\begin{array}{l}\text { 2nd Constituent } \\
\text { Frequency* }\end{array}$} \\
\hline & $H$ & $\%$ & & & & & & \\
\hline $\mathrm{H}$ & 1 & & & & & & & \\
\hline$\%$ & $-.88^{*}$ & 1 & & & & & & \\
\hline Familiarity & $-.26^{*}$ & $.31 *$ & 1 & & & & & \\
\hline Im. agree. & $-.49 *$ & $.52 *$ & $.29 *$ & 1 & & & & \\
\hline Complex. & .05 & -.02 & $-.20 *$ & -.09 & 1 & & & \\
\hline Surf. frq. & -.18 & .17 & .16 & 0 & -.03 & 1 & & \\
\hline 1st c. frq. & -.01 & -.03 & .12 & -.03 & $-.22 *$ & .14 & 1 & \\
\hline 2nd c. frq. & -.12 & .17 & .18 & .13 & .01 & .13 & -.09 & 1 \\
\hline
\end{tabular}

Note. Im. Agree., image agreement; Complex, complexity; Surf. frq., surface frequency; 1st c. frq., 1st constituent frequency; 2nd c. frq., 2nd constituent frequency

*Correlation coefficients significant at $p<.01$ 


\section{Appendix 1}

Table 5 "Item" represents an item number that is consistent between Appendices 1 and 2; "Modal Name" refers to the most common name for the picture on the basis of the participants' responses; "Celex Name" refers to the name of the picture as listed in the CELEX lexical database (Baayen, Piepenbrock, \& van Rijn, 1993), and "na" when it was not listed; "Type" refers to the compound type as listed in
CELEX; $H$ and $\%$ are two measures of name agreement; mean $(M)$ and standard deviation $(S D)$ are listed for the variables familiarity, image agreement, and visual complexity. "Surf. Frq.," "1 Con. Frq.," and " 2 Con. Frq." refer to the frequency estimates from CELEX for the surface and first- and second-constituent lemma frequencies

\begin{tabular}{|c|c|c|c|c|c|c|c|c|c|c|c|c|c|c|c|}
\hline \multirow[t]{2}{*}{ Item } & \multirow[t]{2}{*}{ Modal Name } & \multirow[t]{2}{*}{ Celex Name } & \multirow[t]{2}{*}{ Type } & \multicolumn{2}{|c|}{$\begin{array}{l}\text { Name } \\
\text { Agreement }\end{array}$} & \multicolumn{2}{|c|}{ Familiarity } & \multicolumn{2}{|c|}{$\begin{array}{l}\text { Image } \\
\text { Agreement }\end{array}$} & \multicolumn{2}{|c|}{$\begin{array}{l}\text { Visual } \\
\text { Complexity }\end{array}$} & \multirow[t]{2}{*}{$\begin{array}{l}\text { Surf. } \\
\text { Frq. }\end{array}$} & \multirow[t]{2}{*}{$\begin{array}{l}1 \text { Con. } \\
\text { Frq. }\end{array}$} & \multirow[t]{2}{*}{$\begin{array}{l}2 \text { Con. } \\
\text { Frq }\end{array}$} & \multirow[t]{2}{*}{$\begin{array}{l}\text { Reaction } \\
\text { Time }\end{array}$} \\
\hline & & & & $H$ & $\%$ & $M$ & $S D$ & $M$ & $S D$ & $M$ & $S D$ & & & & \\
\hline 1 & airmattress & air mattress & $\mathrm{NN}$ & 2.22 & 3.33 & 3.44 & 1.74 & 4.97 & 1.29 & 3.23 & 1.24 & 0.00 & 8.41 & 5.48 & 1,399 \\
\hline 2 & airplane & airplane & $\mathrm{NN}$ & 0.05 & 96.67 & 5.34 & 1.54 & 5.96 & 1.02 & 4.35 & 1.54 & 4.30 & 8.41 & 7.04 & 886 \\
\hline 3 & alarmclock & alarm-clock & $\mathrm{NN}$ & 0.60 & 86.67 & 5.58 & 1.58 & 4.95 & 1.71 & 4.55 & 1.49 & 0.69 & 5.96 & 6.56 & 1,293 \\
\hline 4 & ashtray & ashtray & $\mathrm{NN}$ & 1.44 & 43.33 & 2.17 & 1.40 & 4.63 & 1.80 & 2.39 & 1.29 & 4.65 & 6.65 & 6.13 & 1,499 \\
\hline 5 & babycarriage & baby carriage & $\mathrm{NN}$ & 2.06 & 43.33 & 2.74 & 1.43 & 4.39 & 2.22 & 4.18 & 1.59 & 0.00 & 8.44 & 5.64 & 1,218 \\
\hline 6 & backpack & na & $\mathrm{NN}$ & 0.59 & 73.33 & 3.76 & 2.04 & 3.26 & 1.78 & 5.34 & 1.10 & 0.00 & 8.16 & 5.87 & 1,480 \\
\hline 7 & bagpipe & bagpipe & $\mathrm{NN}$ & 0.26 & 80.00 & 1.85 & 1.21 & 3.89 & 1.74 & 5.28 & 1.45 & 1.10 & 7.27 & 6.33 & 1,319 \\
\hline 8 & barbedwire & barbed wire & $\mathrm{AN}$ & 0.91 & 66.67 & 2.28 & 1.51 & 4.45 & 1.63 & 2.57 & 1.22 & 0.00 & 4.98 & 6.67 & 1,211 \\
\hline 9 & baseball & baseball & $\mathrm{NN}$ & 0.05 & 96.67 & 4.18 & 1.73 & 6.55 & 0.71 & 3.25 & 1.44 & 4.71 & 7.27 & 7.60 & 871 \\
\hline 10 & basketball & basketball & $\mathrm{NN}$ & 0 & 100.00 & 4.82 & 1.56 & 6.15 & 0.83 & 2.15 & 0.95 & 3.78 & 6.06 & 7.60 & 872 \\
\hline 11 & bathtub & bathtub & $\mathrm{NN}$ & 0.21 & 96.67 & 5.31 & 1.87 & 4.94 & 1.49 & 2.67 & 0.96 & 3.47 & 6.73 & 5.12 & 1,004 \\
\hline 12 & beachball & beach ball & $\mathrm{NN}$ & 0.46 & 90.00 & 3.33 & 1.34 & 6.07 & 1.04 & 2.09 & 1.20 & 0.00 & 7.28 & 7.60 & 988 \\
\hline 13 & beehive & beehive & $\mathrm{NN}$ & 0.77 & 60.00 & 2.23 & 1.31 & 3.95 & 1.62 & 2.95 & 1.46 & 3.33 & 5.70 & 4.23 & 1,287 \\
\hline 14 & birdcage & bird-cage & $\mathrm{NN}$ & 0.65 & 83.33 & 2.31 & 1.56 & 3.97 & 1.28 & 5.30 & 1.34 & 0.00 & 7.52 & 5.67 & 1,186 \\
\hline 15 & birdhouse & na & $\mathrm{NN}$ & 0.26 & 93.33 & 2.33 & 1.27 & 4.91 & 1.12 & 1.64 & 0.98 & 0.00 & 7.52 & 9.29 & 1,111 \\
\hline 16 & blackberry & blackberry & $\mathrm{AN}$ & 1.67 & 10.00 & 4.05 & 1.72 & 4.06 & 2.10 & 2.69 & 1.16 & 3.30 & 6.97 & 5.23 & 1,826 \\
\hline 17 & blackbird & blackbird & $\mathrm{AN}$ & 2.14 & 16.67 & 3.58 & 2.04 & 5.41 & 1.38 & 5.74 & 1.54 & 3.33 & 6.97 & 7.52 & 1,348 \\
\hline 18 & blackboard & blackboard & $\mathrm{AN}$ & 1.18 & 70.00 & 5.37 & 1.69 & 5.17 & 1.09 & 2.61 & 1.52 & 4.57 & 6.97 & 7.52 & 1,080 \\
\hline 19 & blacksmith & blacksmith & $\mathrm{AN}$ & 2.30 & 36.67 & 1.68 & 1.00 & 4.71 & 1.75 & 6.81 & 0.46 & 3.43 & 6.97 & 3.93 & 1,852 \\
\hline 20 & bluejay & bluejay & $\mathrm{AN}$ & 2.45 & 3.33 & 3.75 & 1.77 & 5.18 & 1.33 & 5.50 & 1.45 & 0.00 & 6.28 & 4.51 & 1,862 \\
\hline 21 & blueprint & blueprint & $\mathrm{AN}$ & 1.38 & 30.00 & 2.88 & 1.90 & 4.25 & 1.71 & 6.15 & 1.38 & 3.99 & 6.28 & 6.18 & 1,653 \\
\hline 22 & bobsled & bobsled & $\mathrm{NN}$ & 1.87 & 56.67 & 2.11 & 1.52 & 4.36 & 2.17 & 6.12 & 0.93 & 0.69 & 4.49 & 3.18 & 1,346 \\
\hline 23 & bookshelf & na & $\mathrm{NN}$ & 1.16 & 56.67 & 6.05 & 1.21 & 4.85 & 1.58 & 3.07 & 1.56 & 0.00 & 8.96 & 6.21 & 1,129 \\
\hline 24 & bowtie & bow tie & $\mathrm{NN}$ & 0.30 & 90.00 & 3.39 & 1.77 & 5.90 & 1.15 & 2.32 & 1.03 & 0.00 & 5.42 & 6.42 & 935 \\
\hline 25 & boxingglove & boxing-glove & $\mathrm{NN}$ & 1.99 & 63.33 & 2.38 & 1.50 & 4.55 & 1.52 & 3.88 & 1.28 & 0.69 & 4.28 & 5.86 & 1,263 \\
\hline 26 & briefcase & briefcase & $\mathrm{NN}$ & 0.58 & 80.00 & 4.18 & 1.24 & 4.46 & 1.79 & 3.96 & 1.03 & 4.98 & 4.20 & 9.09 & 1,262 \\
\hline 27 & bunkbed & bunk bed & $\mathrm{NN}$ & 0.05 & 96.67 & 5.28 & 2.17 & 5.40 & 1.34 & 6.26 & 0.92 & 0.00 & 1.39 & 8.48 & 1,208 \\
\hline 28 & butterfly & butterfly & $\mathrm{NN}$ & 0 & 100.00 & 3.64 & 1.68 & 4.98 & 1.59 & 1.59 & 0.78 & 4.49 & 6.20 & 6.46 & 833 \\
\hline 29 & chainsaw & chain saw & $\mathrm{NN}$ & 1.18 & 66.67 & 1.89 & 1.09 & 5.60 & 1.41 & 4.04 & 1.23 & 0.00 & 6.77 & 3.30 & 1,191 \\
\hline 30 & cheerleader & cheerleader & $\mathrm{VN}$ & 0 & 100.00 & 3.69 & 1.86 & 4.90 & 1.60 & 5.25 & 1.96 & 2.08 & 4.64 & 7.85 & 840 \\
\hline 31 & chessboard & chessboard & $\mathrm{NN}$ & 1.28 & 43.33 & 3.69 & 1.70 & 5.15 & 1.66 & 2.09 & 1.11 & 2.40 & 5.58 & 7.52 & 1,283 \\
\hline 32 & christmastree & christmas-tree & $\mathrm{NN}$ & 0 & 100.00 & 4.29 & 1.86 & 5.40 & 1.37 & 3.63 & 1.52 & 1.39 & 6.98 & 8.14 & 927 \\
\hline 33 & clothespin & clothespin & $\mathrm{NN}$ & 1.30 & 66.67 & 2.90 & 1.22 & 5.37 & 1.92 & 4.16 & 1.50 & 0.00 & 7.71 & 5.70 & 1,523 \\
\hline 34 & corkscrew & corkscrew & $\mathrm{NN}$ & 2.06 & 43.33 & 2.98 & 1.59 & 4.56 & 1.68 & 3.23 & 1.35 & 3.14 & 4.60 & 5.24 & 1,543 \\
\hline 35 & cowboy & cowboy & $\mathrm{NN}$ & 0.26 & 93.33 & 2.11 & 1.35 & 5.32 & 1.49 & 6.07 & 1.22 & 4.44 & 6.58 & 8.74 & 1,319 \\
\hline 36 & creditcard & credit card & $\mathrm{NN}$ & 1.35 & 70.00 & 5.70 & 1.35 & 5.80 & 1.40 & 4.64 & 1.93 & 0.00 & 6.63 & 7.13 & 1,276 \\
\hline 37 & crossbow & crossbow & $\mathrm{NN}$ & 1.37 & 20 & 1.75 & 1.06 & 5.09 & 1.97 & 5.33 & 1.41 & 1.10 & 5.99 & 4.58 & 1,267 \\
\hline 38 & crowbar & crowbar & $\mathrm{NN}$ & 1.67 & 43.33 & 1.91 & 0.91 & 5.56 & 1.63 & 2.57 & 1.82 & 2.48 & 4.53 & 7.41 & 1,403 \\
\hline 39 & cupboard & cupboard & $\mathrm{NN}$ & 2.81 & 0 & 4.79 & 1.72 & 3.50 & 1.67 & 2.07 & 1.04 & 5.87 & 7.23 & 7.52 & na \\
\hline
\end{tabular}


Table 5 (continued)

\begin{tabular}{|c|c|c|c|c|c|c|c|c|c|c|c|c|c|c|c|}
\hline \multirow[t]{2}{*}{ Item } & \multirow[t]{2}{*}{ Modal Name } & \multirow[t]{2}{*}{ Celex Name } & \multirow[t]{2}{*}{ Type } & \multicolumn{2}{|c|}{$\begin{array}{l}\text { Name } \\
\text { Agreement }\end{array}$} & \multicolumn{2}{|c|}{ Familiarity } & \multicolumn{2}{|c|}{$\begin{array}{l}\text { Image } \\
\text { Agreement }\end{array}$} & \multicolumn{2}{|c|}{$\begin{array}{l}\text { Visual } \\
\text { Complexity }\end{array}$} & \multirow[t]{2}{*}{$\begin{array}{l}\text { Surf. } \\
\text { Frq. }\end{array}$} & \multirow[t]{2}{*}{$\begin{array}{l}1 \text { Con. } \\
\text { Frq. }\end{array}$} & \multirow[t]{2}{*}{$\begin{array}{l}2 \text { Con. } \\
\text { Frq }\end{array}$} & \multirow[t]{2}{*}{$\begin{array}{l}\text { Reaction } \\
\text { Time }\end{array}$} \\
\hline & & & & $H$ & $\%$ & $M$ & $S D$ & $M$ & $S D$ & $M$ & $S D$ & & & & \\
\hline 40 & cupcake & cupcake & $\mathrm{NN}$ & 0 & 100.00 & 4.41 & 1.66 & 4.77 & 1.37 & 2.17 & 0.93 & 0.00 & 7.23 & 6.42 & 894 \\
\hline 41 & dartboard & dartboard & $\mathrm{NN}$ & 0.64 & 83.33 & 3.24 & 1.67 & 5.96 & 1.13 & 3.32 & 1.57 & 1.61 & 4.06 & 7.52 & 1,131 \\
\hline 42 & doghouse & doghouse & $\mathrm{NN}$ & 0.26 & 93.33 & 2.38 & 1.45 & 5.73 & 1.75 & 3.52 & 1.11 & 0.00 & 7.63 & 9.29 & 1,211 \\
\hline 43 & doorknob & doorknob & $\mathrm{NN}$ & 0.72 & 76.67 & 5.65 & 1.96 & 4.08 & 1.22 & 2.88 & 1.22 & 2.94 & 8.84 & 4.80 & 1,289 \\
\hline 44 & doughnut & doughnut & $\mathrm{NN}$ & 0 & 100.00 & 4.59 & 1.58 & 5.79 & 1.07 & 3.11 & 0.83 & 3.04 & 5.22 & 6.02 & 922 \\
\hline 45 & dragonfly & dragonfly & $\mathrm{NN}$ & 0.67 & 86.67 & 3.53 & 1.33 & 5.87 & 1.04 & 4.43 & 1.11 & 2.89 & 5.14 & 6.46 & 1,270 \\
\hline 46 & drumstick & drumstick & $\mathrm{NN}$ & 1.65 & 53.33 & 4.39 & 1.80 & 3.72 & 2.36 & 2.39 & 0.98 & 1.39 & 5.65 & 6.55 & 1,478 \\
\hline 47 & dumbbell & dumbbell & $\mathrm{AN}$ & 2.02 & 53.33 & 4.26 & 1.85 & 4.65 & 1.62 & 4.23 & 1.66 & 1.95 & 1.61 & 6.61 & 1,427 \\
\hline 48 & earmuffs & earmuff & $\mathrm{NN}$ & 0.09 & 93.33 & 3.93 & 1.99 & 5.77 & 1.42 & 3.46 & 1.34 & 1.10 & 7.36 & 2.77 & 1,066 \\
\hline 49 & earring & earring & $\mathrm{NN}$ & 0.55 & 83.33 & 4.40 & 2.55 & 4.18 & 2.06 & 3.03 & 1.42 & 2.83 & 7.36 & 6.78 & 1,353 \\
\hline 50 & eggplant & eggplant & $\mathrm{NN}$ & 0.86 & 70.00 & 3.37 & 1.99 & 5.49 & 1.93 & 2.27 & 1.01 & 1.61 & 7.34 & 7.68 & 1,289 \\
\hline 51 & fingerprint & fingerprint & $\mathrm{NN}$ & 0.77 & 83.33 & 3.51 & 1.82 & 6.40 & 1.02 & 5.39 & 1.98 & 2.56 & 7.70 & 6.18 & 1,087 \\
\hline 52 & firecracker & firecracker & $\mathrm{NN}$ & 1.71 & 43.33 & 2.22 & 1.29 & 4.50 & 2.11 & 2.25 & 1.38 & 1.61 & 7.97 & 3.76 & 1,224 \\
\hline 53 & firehose & fire-hose & $\mathrm{NN}$ & 1.05 & 3.33 & 2.72 & 1.40 & 4.40 & 1.69 & 2.64 & 1.17 & 0.00 & 7.97 & 4.29 & 1,412 \\
\hline 54 & fireplace & fireplace & $\mathrm{NN}$ & 0.30 & 90.00 & 4.25 & 1.98 & 4.30 & 1.53 & 2.83 & 1.71 & 5.00 & 7.97 & 9.35 & 1,067 \\
\hline 55 & fishbone & fishbone & $\mathrm{NN}$ & 2.02 & 36.67 & 2.70 & 1.63 & 4.99 & 1.77 & 3.18 & 1.10 & 1.61 & 7.98 & 7.13 & 1,328 \\
\hline 56 & fishinghook & na & $\mathrm{NN}$ & 1.37 & 46.67 & 2.34 & 0.94 & 5.82 & 1.21 & 1.60 & 0.81 & 0.00 & 6.80 & 6.51 & 1,331 \\
\hline 57 & fishingrod & fishing-rod & $\mathrm{VN}$ & 1.37 & 40.00 & 2.31 & 1.11 & 5.54 & 1.10 & 3.41 & 1.42 & 0.69 & 6.80 & 6.50 & 1,504 \\
\hline 58 & flyswatter & flyswatter & $\mathrm{NN}$ & 0.85 & 80.00 & 2.57 & 1.48 & 5.60 & 1.40 & 2.65 & 1.28 & 0.69 & 6.46 & 1.61 & 1,426 \\
\hline 59 & football & football & $\mathrm{NN}$ & 0 & 100.00 & 4.37 & 1.90 & 6.41 & 0.79 & 2.37 & 1.02 & 6.35 & 8.68 & 7.60 & 786 \\
\hline 60 & footprint & footprint & $\mathrm{NN}$ & 0.51 & 86.67 & 4.04 & 1.93 & 5.50 & 1.54 & 1.77 & 1.01 & 2.40 & 8.68 & 6.18 & 916 \\
\hline 61 & fryingpan & frying-pan & $\mathrm{NN}$ & 1.63 & 53.33 & 4.91 & 1.66 & 5.92 & 1.72 & 3.62 & 1.45 & 2.48 & 5.95 & 6.19 & 1,049 \\
\hline 62 & gasmask & gasmask & $\mathrm{NN}$ & 0.91 & 80.00 & 1.52 & 0.83 & 4.70 & 1.97 & 3.78 & 1.28 & 1.79 & 7.23 & 5.87 & 1,232 \\
\hline 63 & gokart & go-kart & $\mathrm{VN}$ & 1.84 & 43.33 & 1.79 & 0.85 & 4.20 & 2.19 & 6.91 & 0.30 & 2.08 & 6.08 & 5.25 & 1,711 \\
\hline 64 & greenhouse & greenhouse & $\mathrm{NN}$ & 2.46 & 30.00 & 2.68 & 1.64 & 4.93 & 1.31 & 3.57 & 1.29 & 4.60 & 6.72 & 9.29 & 1,620 \\
\hline 65 & hairbrush & hairbrush & $\mathrm{NN}$ & 1.40 & 63.33 & 5.09 & 1.58 & 4.59 & 1.68 & 4.09 & 1.42 & 2.20 & 8.18 & 5.69 & 1,050 \\
\hline 66 & handcuffs & handcuff & $\mathrm{NN}$ & 0.09 & 93.33 & 2.39 & 1.64 & 6.36 & 0.88 & 2.83 & 1.22 & 3.56 & 9.47 & 2.64 & 966 \\
\hline 67 & handgrenade & hand-grenade & $\mathrm{NN}$ & 0.88 & 20.00 & 1.84 & 1.32 & 5.20 & 2.09 & 3.67 & 1.13 & 0.69 & 9.47 & 4.99 & 1,257 \\
\hline 68 & handlebars & handlebar & $\mathrm{NN}$ & 2.37 & 33.33 & 4.73 & 1.71 & 4.24 & 1.40 & 3.91 & 1.80 & 1.95 & 5.84 & 7.41 & 1,354 \\
\hline 69 & hangglider & hang-glider & $\mathrm{VN}$ & 1.71 & 56.67 & 1.69 & 0.60 & 5.16 & 1.52 & 5.36 & 1.06 & 1.39 & 2.64 & 4.32 & 1,271 \\
\hline 70 & haystack & haystack & $\mathrm{NN}$ & 1.53 & 60.00 & 1.76 & 0.86 & 4.07 & 1.88 & 4.15 & 1.78 & 2.89 & 5.59 & 5.40 & 1,292 \\
\hline 71 & headlight & headlight & $\mathrm{NN}$ & 1.31 & 73.33 & 4.56 & 1.69 & 3.90 & 2.03 & 4.60 & 1.50 & 3.04 & 9.13 & 8.56 & 1,390 \\
\hline 72 & headphones & headphones & $\mathrm{NN}$ & 0.40 & 90.00 & 6.05 & 1.24 & 4.89 & 1.35 & 4.84 & 1.33 & 3.14 & 9.13 & 7.02 & 1,227 \\
\hline 73 & highchair & high chair & AN & 1.50 & 63.33 & 2.32 & 1.42 & 5.55 & 1.28 & 5.58 & 1.45 & 0.00 & 4.95 & 7.80 & 1,159 \\
\hline 74 & hotdog & hot dog & AN & 0.05 & 96.67 & 5.02 & 1.53 & 5.70 & 1.05 & 3.50 & 1.28 & 0.00 & 7.86 & 7.63 & 824 \\
\hline 75 & hourglass & hourglass & $\mathrm{NN}$ & 0.96 & 56.67 & 2.73 & 1.75 & 5.63 & 1.29 & 2.42 & 1.27 & 2.40 & 8.82 & 7.86 & 1,114 \\
\hline 76 & hovercraft & hovercraft & $\mathrm{VN}$ & 1.48 & 23.33 & 1.71 & 1.23 & 3.19 & 1.94 & 6.24 & 0.85 & 2.08 & 5.37 & 5.78 & 2,171 \\
\hline 77 & icecream & ice-cream & $\mathrm{NN}$ & 0.46 & 90.00 & 5.50 & 1.31 & 6.05 & 1.19 & 3.17 & 1.33 & 4.38 & 6.85 & 6.29 & 848 \\
\hline 78 & iceskate & ice-skate & $\mathrm{NN}$ & 0.26 & 93.33 & 3.16 & 1.92 & 5.81 & 1.14 & 3.69 & 1.26 & 0.69 & 6.85 & 4.33 & 1,098 \\
\hline 79 & ironingboard & ironing-board & $\mathrm{NN}$ & 0.05 & 96.67 & 3.78 & 1.84 & 6.03 & 1.03 & 2.64 & 1.26 & 1.61 & 4.23 & 7.52 & 1,177 \\
\hline 80 & jellyfish & jellyfish & $\mathrm{NN}$ & 0.98 & 76.67 & 2.00 & 1.16 & 5.54 & 1.33 & 3.88 & 1.49 & 2.94 & 5.27 & 7.98 & 1,177 \\
\hline 81 & keyboard & keyboard & $\mathrm{NN}$ & 0.05 & 96.67 & 6.86 & 0.44 & 5.67 & 1.90 & 3.96 & 1.69 & 3.87 & 7.34 & 7.52 & 963 \\
\hline 82 & keyhole & keyhole & $\mathrm{NN}$ & 1.11 & 60.00 & 5.27 & 1.58 & 4.85 & 1.43 & 2.31 & 1.14 & 2.71 & 7.34 & 7.40 & 1,362 \\
\hline 83 & ladybug & ladybug & $\mathrm{NN}$ & 0.21 & 96.67 & 3.61 & 1.43 & 4.80 & 1.49 & 2.47 & 0.95 & 0.00 & 7.61 & 4.48 & 1,093 \\
\hline 84 & lamppost & lamppost & $\mathrm{NN}$ & 2.05 & 46.67 & 4.23 & 1.55 & 4.05 & 2.12 & 5.70 & 1.54 & 3.09 & 6.45 & 7.34 & 1,360 \\
\hline 85 & lifesaver & life-saver & $\mathrm{NN}$ & 2.74 & 26.67 & 2.33 & 1.17 & 3.87 & 2.28 & 3.84 & 1.26 & 1.61 & 9.61 & 3.97 & 1,949 \\
\hline
\end{tabular}


Table 5 (continued)

\begin{tabular}{|c|c|c|c|c|c|c|c|c|c|c|c|c|c|c|c|}
\hline \multirow[t]{2}{*}{ Item } & \multirow[t]{2}{*}{ Modal Name } & \multirow[t]{2}{*}{ Celex Name } & \multirow[t]{2}{*}{ Type } & \multicolumn{2}{|c|}{$\begin{array}{l}\text { Name } \\
\text { Agreement }\end{array}$} & \multicolumn{2}{|c|}{ Familiarity } & \multicolumn{2}{|c|}{$\begin{array}{l}\text { Image } \\
\text { Agreement }\end{array}$} & \multicolumn{2}{|c|}{$\begin{array}{l}\text { Visual } \\
\text { Complexity }\end{array}$} & \multirow[t]{2}{*}{$\begin{array}{l}\text { Surf. } \\
\text { Frq. }\end{array}$} & \multirow[t]{2}{*}{$\begin{array}{l}1 \text { Con. } \\
\text { Frq. }\end{array}$} & \multirow[t]{2}{*}{$\begin{array}{l}2 \text { Con. } \\
\text { Frq }\end{array}$} & \multirow[t]{2}{*}{$\begin{array}{l}\text { Reaction } \\
\text { Time }\end{array}$} \\
\hline & & & & $H$ & $\%$ & $M$ & $S D$ & $M$ & $S D$ & $M$ & $S D$ & & & & \\
\hline 86 & lifevest & na & $\mathrm{NN}$ & 1.86 & 23.33 & 3.31 & 1.81 & 5.03 & 1.38 & 2.83 & 1.10 & 0.00 & 9.61 & 4.89 & 1,136 \\
\hline 87 & lightbulb & lightbulb & $\mathrm{NN}$ & 0.21 & 96.67 & 6.24 & 1.10 & 6.75 & 0.45 & 3.96 & 1.49 & 0.00 & 8.56 & 5.34 & 843 \\
\hline 88 & lighthouse & lighthouse & $\mathrm{NN}$ & 0.40 & 90.00 & 2.66 & 1.33 & 5.33 & 1.20 & 3.96 & 1.84 & 3.87 & 8.56 & 9.29 & 1,307 \\
\hline 89 & lipstick & lipstick & $\mathrm{NN}$ & 0 & 100.00 & 3.58 & 2.36 & 6.16 & 0.75 & 4.33 & 1.38 & 4.81 & 7.25 & 6.55 & 982 \\
\hline 90 & magnifyingglass & $\begin{array}{l}\text { magnifying } \\
\text { glass }\end{array}$ & $\mathrm{VN}$ & 0.46 & 90.00 & 2.73 & 0.99 & 6.24 & 0.88 & 4.14 & 1.51 & 0.00 & 4.85 & 7.86 & 979 \\
\hline 91 & mailbox & mailbox & $\mathrm{NN}$ & 0.05 & 96.67 & 4.82 & 1.44 & 5.58 & 1.47 & 3.50 & 1.10 & 3.22 & 6.13 & 7.52 & 804 \\
\hline 92 & mapleleaf & maple-leaf & $\mathrm{NN}$ & 1.35 & 66.67 & 4.46 & 2.01 & 5.58 & 1.41 & 1.66 & 1.15 & 0.00 & 4.19 & 7.28 & 1,170 \\
\hline 93 & matchbox & matchbox & $\mathrm{NN}$ & 1.59 & 53.33 & 3.98 & 1.79 & 5.50 & 1.54 & 4.18 & 1.60 & 2.64 & 6.93 & 8.96 & 1,145 \\
\hline 94 & motorcycle & motorcycle & $\mathrm{NN}$ & 0.05 & 96.67 & 3.70 & 1.56 & 5.88 & 1.13 & 5.81 & 1.65 & 5.41 & 5.55 & 6.33 & 1,001 \\
\hline 95 & mousetrap & mousetrap & $\mathrm{NN}$ & 0.26 & 93.33 & 2.51 & 1.72 & 5.49 & 1.51 & 5.53 & 1.31 & 2.20 & 5.80 & 6.04 & 1,216 \\
\hline 96 & nailfile & nail-file & $\mathrm{NN}$ & 1.72 & 40.00 & 4.15 & 2.04 & 4.64 & 1.85 & 3.59 & 1.44 & 0.69 & 6.10 & 6.53 & 1,654 \\
\hline 97 & necklace & necklace & $\mathrm{NN}$ & 0.81 & 80.00 & 4.36 & 2.03 & 5.01 & 2.48 & 2.08 & 0.93 & 3.74 & 7.26 & 5.42 & 1,088 \\
\hline 98 & nervecell & nerve-cell & $\mathrm{NN}$ & 1.77 & 6.67 & 3.14 & 1.40 & 5.11 & 1.63 & 2.83 & 1.35 & 0.00 & 5.79 & 7.10 & 1,251 \\
\hline 99 & newspaper & newspaper & $\mathrm{NN}$ & 0 & 100.00 & 5.91 & 1.38 & 5.23 & 1.11 & 4.55 & 1.57 & 7.02 & 7.64 & 8.30 & 821 \\
\hline 100 & notebook & notebook & $\mathrm{NN}$ & 0.67 & 86.67 & 6.18 & 1.45 & 5.06 & 1.64 & 3.57 & 1.24 & 4.93 & 7.64 & 8.96 & 1,097 \\
\hline 101 & notepad & na & $\mathrm{NN}$ & 1.14 & 56.67 & 5.85 & 1.17 & 5.23 & 1.41 & 3.13 & 1.29 & 0.00 & 7.64 & 5.62 & 1,424 \\
\hline 102 & oillamp & na & $\mathrm{NN}$ & 3.23 & 3.33 & 1.76 & 1.39 & 2.90 & 1.67 & 3.42 & 1.08 & 0.00 & 7.74 & 6.45 & 2,039 \\
\hline 103 & padlock & padlock & $\mathrm{NN}$ & 0.69 & 16.67 & 4.96 & 1.50 & 4.13 & 1.14 & 3.17 & 1.18 & 3.22 & 5.62 & 5.63 & 1,447 \\
\hline 104 & paperclip & paper-clip & $\mathrm{NN}$ & 0.34 & 86.67 & 5.38 & 1.56 & 6.78 & 1.08 & 2.13 & 1.24 & 0.69 & 8.30 & 4.19 & 957 \\
\hline 105 & peanut & peanut & $\mathrm{NN}$ & 0 & 100.00 & 4.35 & 1.37 & 5.94 & 0.74 & 3.39 & 1.04 & 3.91 & 5.18 & 6.02 & 890 \\
\hline 106 & pineapple & pinneapple & $\mathrm{NN}$ & 0.05 & 96.67 & 3.91 & 0.94 & 6.66 & 0.98 & 5.28 & 1.23 & 3.81 & 5.73 & 6.30 & 746 \\
\hline 107 & pitchfork & pitchfork & $\mathrm{VN}$ & 0.72 & 66.67 & 2.13 & 1.71 & 4.49 & 1.52 & 2.21 & 1.26 & 0.69 & 5.89 & 5.59 & 1,149 \\
\hline 108 & pooltable & na & $\mathrm{NN}$ & 1.41 & 73.33 & 4.33 & 1.87 & 4.45 & 1.67 & 3.59 & 1.31 & 0.00 & 6.60 & 8.34 & 1,203 \\
\hline 109 & powerstrip & na & $\mathrm{NN}$ & 2.14 & 10.00 & 3.78 & 1.72 & 4.61 & 1.84 & 4.48 & 1.04 & 0.00 & 8.73 & 6.08 & 1,371 \\
\hline 110 & punchingbag & punching bag & $\mathrm{VN}$ & 0.98 & 66.67 & 2.63 & 1.39 & 5.53 & 1.46 & 4.12 & 1.82 & 0.00 & 5.23 & 7.27 & 1,504 \\
\hline 111 & rainbow & rainbow & $\mathrm{NN}$ & 0 & 100.00 & 3.21 & 1.66 & 5.17 & 1.64 & 3.79 & 0.66 & 4.62 & 7.16 & 4.58 & 983 \\
\hline 112 & raindrop & raindrop & $\mathrm{NN}$ & 2.83 & 33.33 & 4.71 & 1.97 & 5.33 & 1.98 & 1.19 & 0.93 & 2.08 & 7.16 & 6.38 & 1,219 \\
\hline 113 & razorblade & razor-bade & $\mathrm{NN}$ & 1.25 & 10.00 & 2.21 & 1.18 & 3.19 & 2.21 & 2.27 & 1.48 & 2.48 & 5.06 & 5.98 & 1,487 \\
\hline 114 & reindeer & reindeer & $\mathrm{NN}$ & 1.72 & 16.67 & 2.12 & 1.69 & 4.72 & 1.18 & 4.73 & 1.42 & 1.10 & 4.53 & 5.35 & 1,601 \\
\hline 115 & rockingchair & rocking-chair & $\mathrm{VN}$ & 0 & 100.00 & 3.91 & 1.84 & 6.06 & 1.51 & 5.20 & 1.58 & 1.79 & 5.53 & 7.80 & 951 \\
\hline 116 & rollercoaster & roller coaster & $\mathrm{NN}$ & 0.09 & 93.33 & 3.90 & 1.35 & 4.47 & 1.62 & 5.63 & 1.08 & 0.00 & 4.54 & 3.04 & 1,231 \\
\hline 117 & rollerskate & roller-skate & $\mathrm{NN}$ & 1.62 & 60.00 & 1.85 & 1.04 & 2.65 & 1.73 & 4.60 & 1.47 & 0.69 & 4.54 & 4.33 & 1,273 \\
\hline 118 & sailboat & sailboat & $\mathrm{VN}$ & 0.26 & 93.33 & 3.16 & 1.79 & 5.13 & 1.07 & 4.82 & 1.42 & 1.10 & 4.56 & 7.22 & 987 \\
\hline 119 & seagull & seagull & $\mathrm{NN}$ & 1.63 & 53.33 & 3.56 & 1.70 & 4.90 & 1.68 & 5.54 & 1.12 & 2.94 & 8.04 & 4.42 & 1,530 \\
\hline 120 & seahorse & seahorse & $\mathrm{NN}$ & 0.09 & 93.33 & 1.81 & 1.66 & 5.97 & 1.16 & 5.21 & 1.11 & 0.00 & 8.04 & 7.77 & 1,032 \\
\hline 121 & seatbelt & seat-belt & $\mathrm{NN}$ & 0.09 & 93.33 & 5.55 & 1.60 & 5.10 & 1.41 & 3.53 & 1.26 & 1.79 & 7.57 & 6.18 & 1,124 \\
\hline 122 & sewingmachine & $\begin{array}{l}\text { sewing- } \\
\text { machine }\end{array}$ & $\mathrm{NN}$ & 0.09 & 93.33 & 2.68 & 1.43 & 5.40 & 1.15 & 4.57 & 1.51 & 0.69 & 4.30 & 7.70 & 1,079 \\
\hline 123 & slotmachine & slot-machine & $\mathrm{NN}$ & 1.85 & 46.67 & 2.66 & 2.01 & 4.97 & 1.29 & 4.13 & 1.68 & 0.69 & 4.63 & 7.70 & 1,523 \\
\hline 124 & snowflake & snowflake & $\mathrm{NN}$ & 0.26 & 93.33 & 4.67 & 1.26 & 5.48 & 1.41 & 3.98 & 1.29 & 1.79 & 7.01 & 4.83 & 857 \\
\hline 125 & snowman & snowman & $\mathrm{NN}$ & 0.05 & 96.67 & 3.78 & 1.79 & 6.40 & 0.84 & 3.41 & 1.16 & 2.08 & 7.01 & 10.28 & 815 \\
\hline 126 & spaceship & spaceship & $\mathrm{NN}$ & 1.81 & 30.00 & 1.32 & 0.81 & 2.39 & 1.80 & 5.73 & 1.49 & 3.47 & 7.79 & 7.17 & 1,416 \\
\hline 127 & spinningwheel & $\begin{array}{l}\text { spinning- } \\
\text { wheel }\end{array}$ & $\mathrm{VN}$ & 1.92 & 26.67 & 1.62 & 0.77 & 4.29 & 2.23 & 4.72 & 1.12 & 1.79 & 6.13 & 6.68 & 1,504 \\
\hline 128 & stoplight & stop light & $\mathrm{NN}$ & 1.11 & 70.00 & 5.86 & 1.66 & 6.14 & 1.01 & 4.53 & 1.51 & 0.00 & 6.87 & 8.56 & 1,358 \\
\hline 129 & strawberry & strawberry & $\mathrm{NN}$ & 0.05 & 96.67 & 5.08 & 1.53 & 5.73 & 1.14 & 3.75 & 1.62 & 3.93 & 6.09 & 5.23 & 845 \\
\hline 130 & suitcase & suitcase & $\mathrm{NN}$ & 0.97 & 60.00 & 4.54 & 1.37 & 4.79 & 1.78 & 3.08 & 1.01 & 5.40 & 6.84 & 9.09 & 1,142 \\
\hline
\end{tabular}


Table 5 (continued)

\begin{tabular}{|c|c|c|c|c|c|c|c|c|c|c|c|c|c|c|c|}
\hline \multirow[t]{2}{*}{ Item } & \multirow[t]{2}{*}{ Modal Name } & \multirow[t]{2}{*}{ Celex Name } & \multirow[t]{2}{*}{ Type } & \multicolumn{2}{|c|}{$\begin{array}{l}\text { Name } \\
\text { Agreement }\end{array}$} & \multicolumn{2}{|c|}{ Familiarity } & \multicolumn{2}{|c|}{$\begin{array}{l}\text { Image } \\
\text { Agreement }\end{array}$} & \multicolumn{2}{|c|}{$\begin{array}{l}\text { Visual } \\
\text { Complexity }\end{array}$} & \multirow[t]{2}{*}{$\begin{array}{l}\text { Surf. } \\
\text { Frq. }\end{array}$} & \multirow[t]{2}{*}{$\begin{array}{l}1 \text { Con. } \\
\text { Frq. }\end{array}$} & \multirow[t]{2}{*}{$\begin{array}{l}2 \text { Con. } \\
\text { Frq }\end{array}$} & \multirow[t]{2}{*}{$\begin{array}{l}\text { Reaction } \\
\text { Time }\end{array}$} \\
\hline & & & & $H$ & $\%$ & $M$ & $S D$ & $M$ & $S D$ & $M$ & $S D$ & & & & \\
\hline 131 & sunflower & sunflower & $\mathrm{NN}$ & 0.94 & 80.00 & 4.21 & 1.49 & 6.04 & 0.87 & 5.03 & 1.53 & 3.04 & 7.91 & 7.42 & 890 \\
\hline 132 & sunglasses & sunglasses & $\mathrm{NN}$ & 0.26 & 93.33 & 5.25 & 1.94 & 5.35 & 1.24 & 4.54 & 1.07 & 4.01 & 7.91 & 6.35 & 856 \\
\hline 133 & swimmingpool & $\begin{array}{l}\text { swimming- } \\
\text { pool }\end{array}$ & $\mathrm{NN}$ & 1.42 & 26.67 & 3.49 & 1.72 & 3.95 & 1.85 & 5.40 & 1.56 & 2.56 & 4.69 & 6.60 & 1,100 \\
\hline 134 & tapemeasure & tape-measure & NN & 1.39 & 53.33 & 3.15 & 1.32 & 5.36 & 1.14 & 4.03 & 1.03 & 1.10 & 6.41 & 7.18 & 1,244 \\
\hline 135 & teabag & teabag & NN & 0.51 & 86.67 & 4.44 & 1.81 & 5.40 & 1.27 & 1.54 & 0.64 & 1.10 & 7.40 & 7.27 & 1,345 \\
\hline 136 & teapot & teapot & NN & 1.18 & 70.00 & 4.03 & 1.65 & 5.49 & 1.82 & 2.74 & 1.31 & 3.99 & 7.40 & 6.47 & 1,126 \\
\hline 137 & thumbtack & thumbtack & NN & 1.57 & 53.33 & 4.03 & 1.74 & 4.95 & 1.73 & 1.58 & 0.82 & 0.00 & 6.17 & 3.91 & 1,166 \\
\hline 138 & toiletpaper & toilet-paper & NN & 0.26 & 93.33 & 6.49 & 1.00 & 5.84 & 1.64 & 2.41 & 1.19 & 1.10 & 6.23 & 8.30 & 893 \\
\hline 139 & toothbrush & toothbrush & NN & 0 & 100.00 & 6.87 & 0.41 & 6.30 & 0.87 & 3.70 & 1.46 & 3.40 & 7.36 & 5.69 & 863 \\
\hline 140 & toothpaste & toothpaste & NN & 0.63 & 90.00 & 6.07 & 1.20 & 4.90 & 1.14 & 2.33 & 1.28 & 3.53 & 7.36 & 4.53 & 1,170 \\
\hline 141 & totempole & totem-pole & NN & 0.18 & 86.67 & 1.65 & 0.68 & 5.97 & 1.22 & 6.43 & 0.82 & 0.69 & 3.09 & 5.93 & 1,144 \\
\hline 142 & trashcan & na & NN & 0.97 & 80.00 & 5.52 & 1.23 & 5.91 & 1.24 & 3.95 & 1.27 & 0.69 & 4.20 & 5.12 & 886 \\
\hline 143 & vacuumcleaner & $\begin{array}{l}\text { vacuum } \\
\text { cleaner }\end{array}$ & $\mathrm{NN}$ & 1.36 & 50.00 & 4.99 & 1.51 & 5.72 & 1.33 & 5.97 & 0.99 & 0.00 & 5.58 & 5.31 & 911 \\
\hline 144 & vendingmachine & $\begin{array}{l}\text { vending } \\
\text { machine }\end{array}$ & $\mathrm{VN}$ & 0.30 & 90.00 & 5.42 & 1.22 & 5.86 & 1.31 & 6.16 & 1.50 & 0.00 & 2.89 & 7.70 & 1,146 \\
\hline 145 & videotape & na & NN & 2.95 & 6.67 & 4.44 & 1.51 & 5.22 & 1.53 & 2.61 & 1.16 & 2.08 & 4.17 & 4.32 & 1,271 \\
\hline 146 & walkman & na & $\mathrm{NN}$ & 2.13 & 40.00 & 4.44 & 1.60 & 4.61 & 1.71 & 5.73 & 1.25 & 0.00 & 6.87 & 10.28 & 1,749 \\
\hline 147 & watermelon & watermelon & $\mathrm{NN}$ & 0.30 & 90.00 & 4.23 & 1.39 & 4.20 & 1.41 & 2.87 & 1.20 & 1.79 & 9.02 & 4.13 & 1,047 \\
\hline 148 & wheelbarrow & wheelbarrow & $\mathrm{NN}$ & 0.05 & 96.67 & 2.48 & 1.24 & 5.39 & 1.56 & 2.78 & 1.27 & 2.83 & 6.68 & 4.19 & 1,097 \\
\hline 149 & wheelchair & wheelchair & $\mathrm{NN}$ & 0.09 & 93.33 & 2.48 & 1.44 & 4.98 & 1.30 & 3.88 & 1.48 & 3.78 & 6.68 & 7.80 & 969 \\
\hline 150 & windmill & windmill & $\mathrm{NN}$ & 0 & 100.00 & 1.94 & 1.49 & 5.51 & 1.60 & 5.82 & 1.17 & 4.77 & 7.67 & 5.61 & 1,038 \\
\hline
\end{tabular}

\section{Appendix 2}

Table 6 "Item" is an item number that is consistent between Appendix 1 and 2. "Modal Name" is the participants' most common name for a picture; "NR" refers to the number of no responses to a particular picture; and "Nondominant Names" lists the various alternative names given to a picture (with their frequency between parentheses).

\begin{tabular}{llcl}
\hline Item & Modal Name & NR & Nondominant Names \\
\hline 1 & airmattress & 17 & $\begin{array}{c}\text { airconditioner (1), airvent (1), blow up } \\
\text { matress (1), bread (1), inflatable (1), } \\
\text { ironingboard (1), matress (2), plate (1), } \\
\text { radiotor (1), sleeping (1), wash (1) }\end{array}$ \\
2 & airplane & 1 & \\
3 & alarmclock & 1 & clock (2), stopwatch (1) \\
4 & ashtray & 11 & block (1), cupholder (2), cuplholder (1), \\
5 & babycarriage & 1 & $\begin{array}{c}\text { babystroller (4), baby stroller (3), } \\
\text { carriage (5), stroller (4) }\end{array}$ \\
6 & backpack & 6 & suitcase (2) \\
7 & bagpipe & 6 & \\
8 & barbedwire & 8 & knot (1), stitches (1) \\
9 & baseball & 1 &
\end{tabular}

Table 6 (continued)

\begin{tabular}{|c|c|c|c|}
\hline Item & Modal Name & NR & Nondominant Names \\
\hline 11 & bathtub & 0 & tub $(1)$ \\
\hline 12 & beachball & 1 & ball (1), volleyball (1) \\
\hline 13 & beehive & 10 & hivenest (1), honeyhive (1) \\
\hline 14 & birdcage & 0 & cage (5) \\
\hline 15 & birdhouse & 1 & birdfeeder (1) \\
\hline 16 & blackberry & 0 & $\begin{array}{l}\text { berry (1), grapes (1), handgun (1), nut } \\
\text { (1), pinecone (1), pine cone (1), } \\
\text { raspberry (21) }\end{array}$ \\
\hline 17 & blackbird & 1 & $\begin{array}{l}\text { bird (11), cardinal (1), crow (9), raven } \\
(1) \text {, scarecrow (1), sparrow (1) }\end{array}$ \\
\hline 18 & blackboard & 0 & chalkboard (7), map (1), screen (1) \\
\hline 19 & blacksmith & 6 & $\begin{array}{l}\text { anvil (3), carpenter (2), guy hammers } \\
\text { (1), hammer (2), hammer and anvil } \\
\text { (1), iron and hammer (1), iron worker } \\
\text { (1), metalsmith (2) }\end{array}$ \\
\hline 20 & bluejay & 2 & $\begin{array}{l}\text { bird (8), bluebird (6), canary (1), } \\
\text { cardinal (7), robin (3), sparrow (1), } \\
\text { woodpecker (1) }\end{array}$ \\
\hline
\end{tabular}


Table 6 (continued)

\begin{tabular}{llcl}
\hline Item & Modal Name & NR & Nondominant Names \\
\hline 21 & blueprint & 5 & floorplan (13), map (3) \\
22 & bobsled & 3 & $\begin{array}{c}\text { ice luge (3), skiluge (1), sled (2), sledder } \\
(1), \text { sledding (1), snowboard (1), } \\
\end{array}$ \\
& & tobagon (1)
\end{tabular}

$\begin{array}{clll}23 & \text { bookshelf } & 1 & \text { bookcase (11), shelves (1) } \\ 24 & \text { bowtie } & 2 & \text { bow (1) } \\ 25 & \text { boxingglove } & 0 & \begin{array}{c}\text { boxingmit (3), boxing mit (2), } \\ \text { fightingmit (1), glove (1), mit (1), } \\ \end{array} \\ & & \begin{array}{l}\text { mitten (1), ovenmit (1), punching } \\ \text { glove (1) }\end{array}\end{array}$

\begin{tabular}{|c|c|c|}
\hline briefcase & 4 & carryingcase (1), suitcase (1) \\
\hline bunkbed & 1 & \\
\hline chainsaw & 2 & handsaw (1), powersaw (1), saw (6) \\
\hline chessboard & 0 & checkboard (2), checkerboard (13) \\
\hline clothespin & 4 & $\begin{array}{l}\text { clotheshanger }(1) \text {, hairpin }(1) \text {, paper }(1) \text {, } \\
\text { paperclip (2), pin (1) }\end{array}$ \\
\hline corkscrew & 6 & $\begin{array}{l}\text { bottleopener (1), bottle opener (2), } \\
\text { corkopener (2), drill (1), screw (1), } \\
\text { screwdriver (2), wineopener (2) }\end{array}$ \\
\hline cowboy & 1 & lasso (1) \\
\hline creditcard & 1 & bow (1), ID (3), ID card (3), radio (1) \\
\hline crossbow & 5 & arrow (2), bow (1), bow and arrow (16) \\
\hline crowbar & 9 & $\begin{array}{l}\text { cane }(3) \text {, lockpick }(1) \text {, rope }(1), \text { snake } \\
(1), \text { walking cane }(1) \text {, wire }(1)\end{array}$ \\
\hline cupboard & 4 & $\begin{array}{l}\text { armoir (2), briefcase (1), bureau (3), } \\
\text { cabinet (2), chest (2), closet (3), } \\
\text { drawers (1), dresser (4), } \\
\text { dresserdrawers }(1), \text { wardrobe }(7)\end{array}$ \\
\hline dartboard & 2 & bullseye (1), target (2) \\
\hline doghouse & 1 & birdhouse (1) \\
\hline doorknob & 2 & doorhandle (5) \\
\hline dragonfly & 1 & earthfly (1), firefly (1), mosuito (1) \\
\hline drumstick & 2 & $\begin{array}{l}\text { chickenleg (8), chicken leg (1), leg (1), } \\
\text { thigh (1), turkeyleg (1) }\end{array}$ \\
\hline dumbbell & 2 & $\begin{array}{l}\text { barbell (4), blot (1), free weight (1), } \\
\text { hand weight (1), weight (3), weightlift } \\
\text { (1), weights (1) }\end{array}$ \\
\hline
\end{tabular}

\begin{tabular}{|c|c|c|}
\hline earmuffs & 2 & \\
\hline earring & 3 & braces (1), earhook (1) \\
\hline eggplant & 5 & pear (1), plum (3) \\
\hline fingerprint & 0 & thumbprint (4), thumb print (1) \\
\hline firecracker & 3 & $\begin{array}{l}\text { candle (1), dynamite (10), firework (1), } \\
\text { fireworks (1), match (1) }\end{array}$ \\
\hline firehose & 2 & $\begin{array}{l}\text { gardenhose (2), hose (23), waterhose } \\
(1), \text { wateringhose (1) }\end{array}$ \\
\hline fireplace & 2 & fire (1) \\
\hline fishbone & 1 & $\begin{array}{l}\text { dogfish (1), fish (8), fish skeleton (5), } \\
\text { skeleton (4) }\end{array}$ \\
\hline fishinghook & 3 & fishhook (2), hook (11) \\
\hline fishingrod & 2 & $\begin{array}{l}\text { fishinghook (1), fishingline (1), } \\
\text { fishingpole (14) }\end{array}$ \\
\hline flyswatter & 2 & fly (1), mosquito (1), net (2) \\
\hline footprint & 1 & foot (3) \\
\hline
\end{tabular}

Table 6 (continued)

\begin{tabular}{|c|c|c|c|}
\hline Item & Modal Name & NR & Nondominant Names \\
\hline 61 & fryingpan & 3 & $\begin{array}{l}\text { pan (7), pot (1), saucepan (1), sauce pan } \\
(1) \text {, skillet (1) }\end{array}$ \\
\hline 62 & gasmask & 2 & $\begin{array}{l}\text { airmask (1), facemask (1), mask (1), } \\
\text { oxygenmask (1) }\end{array}$ \\
\hline 63 & gokart & 7 & $\begin{array}{l}\text { bumpercar (3), car (1), paddleboat (1), } \\
\text { racecar (3), race car (1), racingcar (1) }\end{array}$ \\
\hline 64 & greenhouse & 1 & $\begin{array}{l}\text { frame (2), frame of a house (1), framing } \\
\text { (1), house (11), plainwood (1), rain } \\
\text { (1), shed (1), structure (1), unfinished } \\
\text { house (1) }\end{array}$ \\
\hline 65 & hairbrush & 1 & brush (6), comb (2), haircomb (2) \\
\hline 66 & handcuffs & 2 & \\
\hline 67 & handgrenade & 5 & grenade (19) \\
\hline 68 & handlebars & 2 & $\begin{array}{l}\text { bicycle (10), bicycle handle (1), bicyle } \\
\text { (1), bikebrakes (1), bike handle (1), } \\
\text { bikehandles (1), bike handles (1), } \\
\text { handles (1), hand thing (1) }\end{array}$ \\
\hline 69 & hangglider & 5 & $\begin{array}{l}\text { delta (1), glider (1), kite (1), parachute } \\
\text { (1), paraglider (1), paragliding (1), } \\
\text { parasail (2) }\end{array}$ \\
\hline 70 & haystack & 4 & $\begin{array}{l}\text { baybail (1), berries (1), hay (2), hut (3), } \\
\text { nest (1) }\end{array}$ \\
\hline 71 & headlight & 2 & $\begin{array}{l}\text { bumper (1), carlight (1), fender (1), front } \\
\text { headlight (1), headlamp (1), } \\
\text { rearviewmirror (1) }\end{array}$ \\
\hline 72 & headphones & 1 & earphones (2) \\
\hline 73 & highchair & 3 & $\begin{array}{l}\text { babychair (3), babyseat (2), babystool } \\
\text { (1), chair (1), highseat (1) }\end{array}$ \\
\hline 74 & hotdog & 1 & \\
\hline 75 & hourglass & 10 & time (1), timecapsule (1), timeglass (1) \\
\hline 76 & hovercraft & 16 & $\begin{array}{l}\text { bumpercar (3), bumpercars (1), } \\
\text { dunebuggy (1), hoverboat (1), } \\
\text { racecardriver (1) }\end{array}$ \\
\hline 77 & icecream & 1 & icecream cone (1), rollerskate (1) \\
\hline 78 & iceskate & 1 & skate (1) \\
\hline 79 & ironingboard & 1 & \\
\hline 80 & jellyfish & 2 & octopus (2), squid (2), stingray (1) \\
\hline 81 & keyboard & 1 & \\
\hline 82 & keyhole & 3 & lock (8), plug (1) \\
\hline 83 & ladybug & 0 & bug (1) \\
\hline 84 & lamppost & 1 & $\begin{array}{l}\text { lamp (2), lightpole (2), lightpost (1), } \\
\text { streetlamp (6), streetlight (4) }\end{array}$ \\
\hline 85 & lifesaver & 6 & $\begin{array}{l}\text { buoy (1), innertube (1), life (3), } \\
\text { lifepreserver (1), life preserver (3), } \\
\text { liferaft (2), lifering (1), life ring (1), } \\
\text { life thing (1), preserver (1), rope (1) }\end{array}$ \\
\hline 86 & lifevest & 2 & $\begin{array}{l}\text { jacket (1), lifejacket (8), life preserver } \\
(1) \text {, vest (11) }\end{array}$ \\
\hline 87 & lightbulb & 0 & light (1) \\
\hline 88 & lighthouse & 1 & lighttower (2) \\
\hline 90 & magnifyingglass & 1 & microscope (1), spyglass (1) \\
\hline 91 & mailbox & 1 & \\
\hline 92 & mapleleaf & 0 & canada (3), canadianleaf (1), leaf (6) \\
\hline
\end{tabular}


Table 6 (continued)

\begin{tabular}{l}
\hline Item Modal Name $\quad$ NR Nondominant Names \\
\hline 93
\end{tabular}

\begin{tabular}{|c|c|c|c|}
\hline 93 & matchbox & 1 & $\begin{array}{l}\text { matchbook (2), matches (9), } \\
\text { mtachbookbox (1), table (1) }\end{array}$ \\
\hline 94 & motorcycle & 1 & \\
\hline 95 & mousetrap & 1 & mousecatcher (1) \\
\hline 96 & nailfile & 3 & $\begin{array}{l}\text { butter knife (1), file (3), knife (10), } \\
\text { penknife (1) }\end{array}$ \\
\hline 97 & necklace & 1 & pearl necklace (4), pearls (1) \\
\hline 98 & nervecell & 11 & $\begin{array}{l}\text { axon (1), bloodvessel (1), cell (1), nerve } \\
(1), \text { neuron (11), spider web (1), } \\
\text { synapse (1) }\end{array}$ \\
\hline 100 & notebook & 1 & addressbook (1), book (1), journal (1) \\
\hline 101 & notepad & 4 & notebook (8), spiral notebook (1) \\
\hline 102 & oillamp & 4 & $\begin{array}{l}\text { bottle (1), genie (1), genie bottle (1), } \\
\text { genielamp (4), genie lamp (1), kettle } \\
\text { (1), lamp (4), lantern (1), magiclamp } \\
\text { (1), magic lamp (3), teakettle (2), tea } \\
\text { kettle (1), teapot (4) }\end{array}$ \\
\hline
\end{tabular}

103 padlock 1 lock (24)

104 paperclip 3 pin (1)

106 pineapple

107 pitchfork

108 pooltable

109 powerstrip

110 punchingbag

112 raindrop

113 razorblade

114 reindeer

116 rollercoaster

117 rollerskate

118 sailboat

119 seagull

120 seahorse

121 seatbelt

122 sewingmachine 2

123 slotmachine

124 snowflake

125 snowman

126 spaceship

127 spinningwheel
Table 6 (continued)

\begin{tabular}{|c|c|c|c|}
\hline Item & Modal Name & NR & Nondominant Names \\
\hline & & & spinningmachine (1), wheel (1) \\
\hline 128 & strawberry & 1 & \\
\hline 129 & suitcase & 0 & briefcase (12) \\
\hline 130 & sunflower & 1 & daisy (2), dandelion (1), flower (2) \\
\hline 131 & sunglasses & 1 & aviators (1) \\
\hline 132 & swimmingpool & 3 & lake (2), patio (1), pool (16) \\
\hline 133 & tapemeasure & 1 & measuringtape (9), measuring tape (4) \\
\hline 134 & teabag & 2 & pricetag (1), tag (1) \\
\hline 135 & teapot & 0 & teacup (1), teakettle (7), tea kettle (1) \\
\hline 136 & thumbtack & 2 & pin (3), pushpin (2), tack (7) \\
\hline 137 & toiletpaper & 1 & toilet paper roll (1) \\
\hline 139 & toothpaste & 0 & ointment (1), paste (1), tooth (1) \\
\hline 140 & totempole & 4 & \\
\hline 141 & stoplight & 0 & stoplight (21), streetlight (2) \\
\hline 142 & trashcan & 0 & garbagebin (1), garbage can (4), trashbin (1) \\
\hline 143 & vacuumcleaner & 2 & vaccum (3), vacuum (10) \\
\hline 144 & vendingmachine & 2 & snackmachine (1) \\
\hline 145 & videotape & 0 & $\begin{array}{l}\text { cassette (2), cassette tape (2), CD tape } \\
\text { (1), tape (4), taperecorder (1), VHS } \\
\text { (1), vhscassette (1), video (1), } \\
\text { videocassette (5) }\end{array}$ \\
\hline 146 & walkman & 7 & $\begin{array}{l}\text { cassetteplayer (2), cassette player (2), } \\
\text { headphones (2), radio (1), stereo (1), } \\
\text { tapeplayer (1), video (1), videotape (1) }\end{array}$ \\
\hline 147 & watermelon & 2 & cantelope (1) \\
\hline 148 & wheelbarrow & 1 & \\
\hline 149 & wheelchair & 2 & \\
\hline
\end{tabular}

\section{References}

Alario, F.-X., \& Ferrand, L. (1999). A set of 400 pictures standardized for French: Norms for name agreement, image agreement, familiarity, visual complexity, image variability, and age of acquisition. Behavior Research Methods, Instruments, \& Computers, 31, 531-552.

Alario, F.-X., Ferrand, L., Laganaro, M., New, B., Frauenfelder, U. H., \& Segui, J. (2004). Predictors of picture naming speed. Behavior Research Methods, Instruments, \& Computers, 36, 140-155.

ArtExplosion, (1998). NOVA Development [CD-ROM].

Baayen, R. H. (2008). Analyzing linguistic data. A practical introduction to statistics using $R$. Cambridge: Cambridge University Press.

Baayen, R. H., Piepenbrock, R., \& van Rijn, A. (1993). The CELEX lexical database [CD-ROM]. Philadelphia: University of Pennsylvania, Linguistic Data Consortium.

Badecker, W. (2001). Lexical composition and the production of compounds: Evidence from neologistic errors in naming. Language and Cognitive Processes, 16, 337-366.

Balota, D. A., Pilotti, M., \& Cortese, M. J. (2001). Subjective frequency estimates for 2,938 monosyllabic words. Memory \& Cognition, 29, 639-647.

Barry, C., Morisson, C. M., \& Ellis, A. W. (1997). Naming the Snodgrass and Vanderwart pictures: Effects of age of acquisition, frequency, and name agreement. Quarterly Journal of Experimental Psychology, 50A, 560-585. 
Bien, H., Levelt, W. J. M., \& Baayen, H. (2005). Frequency effects in compound production. Proceedings of the National Academy of Sciences, 102, 17876-17881.

Cycowicz, Y. M., Friedman, D., Rothstein, M., \& Snodgrass, J. G. (1997). Picture naming by young children: Norms for name agreement, familiarity and visual complexity. Journal of Experimental Child Psychology, 65, 171-237.

Dimitropoulou, M., Duñabeitia, J. A., Blitsas, P., \& Carreiras, M. (2009). A standardized set of 260 pictures for modern Greek: Norms for name agreement, age of acquisition, and visual complexity. Behavior Research Methods, 41, 584-589.

Dohmes, P., Bölte, J., \& Zwitserlood, P. (2004). The impact of semantic transparency of morphologically complex words on picture naming. Brain and Language, 90, 203-212.

Ellis, A. W., \& Morrison, C. M. (1998). Real age-of-acquisition effects in lexical retrieval. Journal of Experimental Psychology: Learning, Memory, and Cognition, 24, 515-523.

Forster, K. I., \& Forster, J. C. (2003). DMDX: A Windows display program with millisecond accuracy. Behavior Research Methods, Instruments, \& Computers, 35, 116-124.

Gernsbacher, M. A. (1984). Resolving 20 years of inconsistent interactions between lexical familiarity and orthography, concreteness, and polysemy. Journal of Experimental Psychology: General, 113, 256-281.

Godden, D. R., \& Baddeley, A. D. (1975). Context-dependent memory in two natural environments: On land and underwater. British Journal of Psychology, 66, 325-332.

Goldrick, M., Folk, J., \& Rapp, B. (2010). Mrs. Malaprop's neighborhood: Using word errors to reveal neighborhood structure. Journal of Memory and Language, 62, 113-134.

Gordon, B. (1985). Subjective frequency and the lexical decision latency function: Implications for mechanisms of lexical access. Journal of Memory and Language, 24, 631-645.

Hocking, J., McMahon, K., \& de Zubicaray, G. (2010). Semantic interference in object naming: An fMRI study of the postcue naming paradigm. NeuroImage, 50, 796-801.

Janssen, N., Bi, Y., \& Caramazza, A. (2008). A tale of two frequencies: Determining the speed of lexical access for
Mandarin Chinese and English compounds. Language and Cognitive Processes, 23, 1191-1223.

Joanes, D. N., \& Gill, C. A. (1998). Comparing measures of sample skewness and kurtosis. The Statistician, 47, 183-189.

Lachman, R., Shaffer, J. P., \& Hennrikus, D. (1974). Language and cognition: Effects of stimulus codability, name-word frequency, and age of acquisition on lexical reaction time. Journal of Verbal Learning \& Verbal Behavior, 13, 613-625.

Roelofs, A., \& Baayen, H. (2002). Morphology by itself in planning the production of spoken words. Psychonomic Bulletin \& Review, 9, 132-138.

Sanfeliu, M. C., \& Fernández, A. (1996). A set of 254 Snodgrass-Vanderwart pictures standardized for Spanish: Norms for name agreement, image agreement, familiarity, and visual complexity. Behavior Research Methods, Instruments, \& Computers, 28, 537-555.

Shannon, C. E. (1948). A mathematical theory of communication. Bell System Technical Journal, 27, 379-423.

Snodgrass, J. G., \& Vanderwart, M. (1980). A standardized set of 260 pictures: Norms for name agreement, image agreement, familiarity, and visual complexity. Journal of Experimental Psychology: Human Learning and Memory, 6, 174-215.

Snodgrass, J. G., \& Yuditsky, T. (1996). Naming times for the Snodgrass and Vanderwart pictures. Behavior Research Methods, Instruments, \& Computers, 28, 516-536.

Strijkers, K., Costa, A., \& Thierry, G. (2010). Tracking lexical access in speech production: Electrophysiological correlates of word frequency and cognate effects. Cerebral Cortex, 20, 912-928.

Székely, A., D'amico, S., Devescovi, A., Federmeier, K., Herron, D., Iyer, G., et al. (2003). Timed picture naming: Extended norms and validation against previous studies. Behavior Research Methods, Instruments, \& Computers, 35, 621-633.

Tukey, J. W. (1977). Exploratory data analysis. Reading, MA: Addison-Wesley.

Vitkovitch, M., \& Tyrell, L. (1995). Sources of disagreement in object naming. Quarterly Journal of Experimental Psychology, 48A, $822-848$.

Zipf, G. K. (1932). Selected studies of the principle of relative frequency in language. Cambridge, MA: Harvard University Press. 